\title{
functionalObjects.h: Using Symbolic Syntax in C++ Programs
}

\author{
R. Nolty \\ California Institute of Technology, Pasadena, California 91125
}

\begin{abstract}
Objects.h allows the $\mathrm{C}++$ programmer performing common calculations to use a more symbolic syntax rather than an algorithmic syntax. This is not as ambitious as a symbolic manipulation program such as Mathematica; it is more like having the ability to drop a very simple Mathematica statement into a C++ program.
\end{abstract}

\section{INTRODUCTION}

A physicist is often faced with the task of writing a program to perform a relatively straightforward mathematical manipulation. For example, she may need to multiply a couple of multivariate functions together and integrate over one variable to obtain a new multivariate function. Using FORTRAN or $\mathrm{C}$ or a procedural approach with $\mathrm{C}++$, the resulting code may be several hundred lines long and include calls to CERNlib routines with nonobvious names and calling sequences.

Similarly, a physicist may be reading a piece of procedural code written by someone else, and only after several hours of study be able to verify that the code is indeed performing a simple mathematical function.

Computer Algebra Systems (CAS) such as Mathematica or Maple avoid these problems; they allow the physicist to express the mathematical function to be evaluated rather than the algorithm for evaluating it. However, most of us have most of our analysis paraphernalia and infrastructure in programming languages. Results that are easily obtained in a CAS are often not useful in solving an analysis problem.

This paper documents an initial attempt to use the facilities of $\mathrm{C}++$ to allow a programmer to express mathematical operations more directly in the programming language itself.

\section{WHAT DOES IT LOOK LIKE?}

A program to calculate a double definite integral,

$$
\int_{0}^{1} d y \int_{0}^{y} d x x * y
$$

could be written like this:

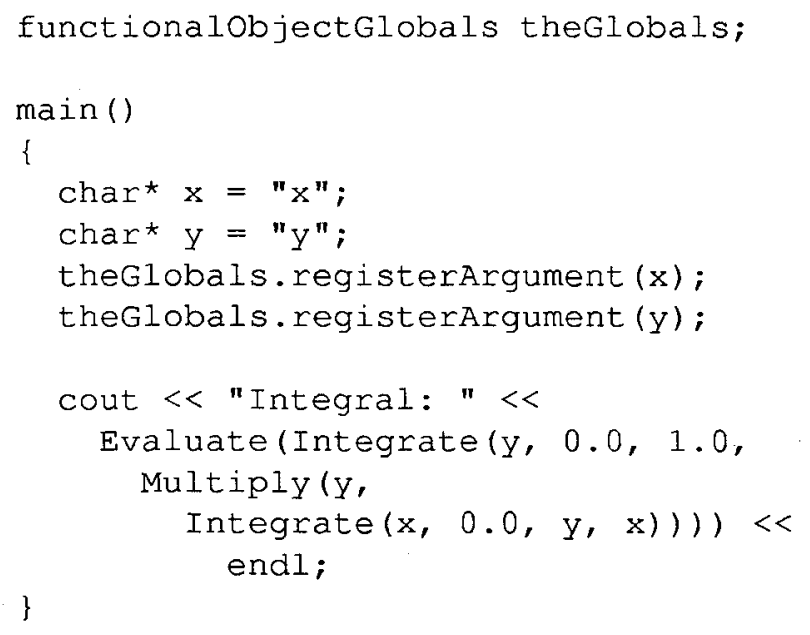

All the action is in the last statement. Reading it from the inside out, Integrate $(\mathrm{x}, 0, \mathrm{y}, \mathrm{x})$ is a $\mathrm{C}++$ function returning a functionalObject which represents the definite integral over $\mathrm{dx}$, with lower limit 0 and upper limit $\mathrm{y}$, of the function x. Multiply(y, Integrate(...)) is a C++ function returning a functionalObject which represents the product of the function $\mathrm{y}$, and the function defined by Integrate(...). Integrate(y,0,1,Multiply(...)) is a $\mathrm{C}++$ function returning a functionalObject which represents the definite integral over dy, with lower limit 0 and upper limit 1 , of the function represented by Multiply(...). Finally, Evaluate(...) is a $\mathrm{C}++$ function which asks its argument, a functionalObject, to evaluate its numeric value and returns that value. Thus, if the program is run, it produces the output

Integral: 0.124994 which differs by roundoff error from the exact result 1/8. 


\section{DISTINCTIVES OF THIS EFFORT}

The effort to date is very preliminary; it has involved only a few days of thinking, a couple of days of coding, and a few hundred lines of $\mathrm{C}++$.

Although functionalObjects.h allows symbolic functions to be expressed, it is not a symbolic manipulation program. For example, the product of one functionalObject representing the function $x$, and another $1 / x$, would not be simplified to 1 . Instead, $x$ would be evaluated, $1 / x$ would be evaluated, and their product would be evaluated. The system is designed to produce numeric results, not symbolic results.

The chief aim is to produce programs that are easy to write, easy to read, and easy to maintain.

\section{IMPLEMENTATION}

The heart of the package is the abstract base class functionalObject, which simply defines a virtual function evaluate(), returning a double. While the evaluate() function takes no arguments, the numerical value of a function may depend on a functionalArgument. For example, a functionalObject may represent $\sin (\mathrm{x})$. If so, the evaluate() function will check the current value of $x$, and return its sine. functionalArguments are managed by a global structure, functionalObjectGlobals. It has functions to declare an argument, to set the value of an argument, and to inquire the current value of an argument.

Often, more than one function may depend on the same argument. For example, a neutrino cross section and a neutrino flux may depend on the same argument, Enu. If they are evaluated at the same time, they will both query the functionalObjectGlobals for the current value of Enu (and any other arguments they depend on).

Application programmers may define classes inheriting from functionalObject to compute arbitrarily complex functions. The package also provides a few commonlyneeded functions; for example, a multiplyObject is a class implementing the product of two functions (each represented by a functionalObject). It simply stores pointers to the two functionalObjects. When the multiplyObject is evaluated, it evaluates its two functions (actually it asks the two functionalObjects to evaluate themselves) and returns the product.

An integrateObject represents a definite integral. When it is asked to evaluate itself, it varies its integration variable (by communication with the functionalObjectGlobals) over its integration range and at each point evaluates its argument, which must be a functionalObject, until it has computed the definite integral.
Programs are made a bit more readable by the existence of certain functions that construct and return functionalObjects. For example, Multiply(functionalObject fcn1, functionalObject fen2) constructs and returns a multiplyObject. Some overloads also implicitly create very simple functions. Mutliply(3.0,fcn1) creates a doubleObject which always evaluates to the double 3.0 , and then returns a multiplyObject that represents the product of the new doubleObject and fen1. Multiply(" $x$ ",fen1) creates an argumentObject that always evaluates to the current value of the functionalArgument $x$, and then returns a multiplyObject that represents the product of the new argumentObject and fen 1 . Similarly, Integrate(char* integrationVariable, functionalObject* lowerLimit, functionalObject* upperLimit, functionalObject* integrand) constructs and returns the appropriate integrateObject representing a definite integral.

\section{SHORTCOMINGS}

As stated above, the effort is not very mature at this point. It exhibits several shortcomings, some of which could be overcome with further effort.

For some problems, a procedural approach could take advantage of peculiarities of the problem to make a much more efficient algorithm. The emphasis here is on ease of programming and ease of reading and maintaining programs, not on efficiency.

In the current implementation, functions that implicitly depend on arguments access their arguments by name. It is a bit like the early days of programming before formal parameters were invented. A function, squareX, that computes the square of $x$, is of no value if you want to compute the square of $y$. This greatly limits the ability of a programmer to develop generic functions or use functions developed by other programmers. This limitation could be overcome by making the evaluate() function accept arguments, which would be the names of functionalArguments on which the function is to depend.

No thought has been given to memory management. Some functions implicitly declare new variables on the heap, but they are never recovered when the functions go out of scope.

The operation of the package could be made more transparent. For example, an argument class could be declared whose constructor would declare the argument to the functionalObjectGlobals. Rather than using the syntax Multiply(fen 1,fen2), an overload of the operator* could be used. However, I am somewhat reluctant to make these changes. They would make the programs appear simpler but it would not really be any simpler. I find that physicists are more comfortable when they can see 
how the package works, rather than having the mechanism hidden by programming gimmicks.

\section{CONCLUSION}

This effort, while very preliminary, shows that $\mathrm{C}++$ has powerful facilities making it possible to express some mathematical operations more directly than has been possible in procedural languages.

The library and some example programs are available at

http://www.hep.caltech.edu/ñolty/functionalObjects/ functionalObjects.tgz

in a gzipped tar archive. No license has been developed, but if there is interest I will place it under the Gnu Public License (GPL). 